\title{
The high burden of inpatient diabetes mellitus: the Melbourne Public Hospitals Diabetes Inpatient Audit
}

\section{Leon A Bach \\ MBBS, PhD, FRACP Deputy Director, Department of Endocrinology and Diabetes, ' and Professor ${ }^{2}$ \\ Elif I Ekinc \\ MBBS, FRACP, PhD Director of Diabetes, ${ }^{3}$ and Senior Research Officer ${ }^{4}$ \\ Dennis Engler MD, FRACP Endocrinologist, ${ }^{5}$ and Adjunct Clinical Associate Professor ${ }^{2}$ \\ Chris Gilfillan MBBS, PhD, FRACP Director of Endocrinology and General Medicine, ${ }^{6}$ and Adjunct Clinical Professor ${ }^{2}$ \\ P Shane Hamblin MBBS(Hons), FRACP Head of Endocrinology? \\ Endocrinologist, and Honorary Associate Professor ${ }^{4}$ \\ Richard J Maclsaac \\ PhD, MBBS, FRACP \\ Director, Department of Endocrinology and Diabetes, 8 and Professor \\ Georgia Soldatos \\ MBBS, FRACP, PhD Deputy Director Diabetes, and Research Fellow}

Cheryl Steele RN RM CDE Manager Diabetes Education

Glenn M Ward MBBS, FRACP, DPhil(Oxon) Deputy Director, Department of Endocrinology and Diabetes, ${ }^{8}$ and Associate Professor ${ }^{4}$

Sue Wyatt

RN, GradCertDiabE Diabetes Education Manager

1 Alfred Health, Melbourne, VIC

2 Monash University, Melbourne, VIC

3 Austin Health, Melbourne, VIC

4 University of Melbourne Melbourne, VIC

5 Monash Health Melbourne, VIC

6 Eastern Health Melbourne, VIC

7 Western Health Melbourne, VIC

8 St Vincent's Hospital, Melbourne, VIC

leon.bach@monash.edu

MJA 2014; 201: 334-338 doi: 10.5694/mjal3.00104 cec he prevalence of diabetes mellitus in the Australian community is high and increasing rapidly. ${ }^{1-3}$ Diabetes and its complications cause substantial morbidity and premature mortality, and account for a significant proportion of total health expenditure. For example, patients with diabetes in the United States account for US\$123 billion or $26 \%$ of inpatient expenditure. 4 The prevalence of diabetes in adult inpatients ranges from 5.8\% to $26.2 \%$ in the US, United Kingdom and Japan, ${ }^{5-7}$ but there are currently no equivalent Australian data. We therefore undertook a survey to determine the prevalence of diabetes in inpatients in 11 hospitals in metropolitan Melbourne, which included community, secondary and tertiary hospitals and one aged care and rehabilitation centre. We compared demographic parameters between patients with and without diabetes, and collected more detailed information about management and complications from patients with diabetes.

\section{Methods}

Point prevalence patient surveys were conducted in 11 Melbourne metropolitan hospitals, each on a single day between 30 November 2010 and 22 November 2012. The study was approved by the human research ethics committees of each centre.

Adult inpatients in all wards except intensive care, emergency, obstetrics and psychiatry were surveyed using a scripted interview. Patients were interviewed unless they were absent, too unwell to respond or insufficiently proficient in English. In these cases, medical records were used. Patients were questioned about diabetes status, family history of diabetes, age $>40$ years and medications (lipid-lowering, antihypertensive and blood-thinning medication and glucocorticoids).

Abstract

Objective: To determine the prevalence of diabetes in inpatients in Melbourne hospitals.

Design: Point prevalence survey of all inpatients in each hospital on a single day between 30 November 2010 and 22 November 2012

Setting: 11 hospitals in metropolitan Melbourne including community, secondary and tertiary hospitals and one aged care and rehabilitation centre.

Participants: 2308 adult inpatients in all wards apart from intensive care, emergency, obstetrics and psychiatry.

Main outcome measures: Point prevalence of self-reported diabetes, details of current medication, self-reported frequency of complications.

Results: Diabetes status was obtained in 2273 of 2308 inpatients (98.5\%). Of these, 562 (24.7\%) had diabetes (95\% Cl, 22.9\%-26.5\%). Diabetes prevalence ranged from $15.7 \%$ to $35.1 \%$ in different hospitals $(P<0.001)$. Patients with diabetes were older, heavier and more likely to be taking lipidlowering, antihypertensive and blood-thinning medications. Of 388 patients with complete medication information, 270 (69.6\%) were taking oral hypoglycaemic agents alone or in combination with insulin, 158 (40.7\%) were treated with insulin (67 [17.3\%] with insulin alone) and 51 (13.1\%) were not taking medication for diabetes. The frequency of diabetes complications was very high: 207/290 (71.4\%) for any microvascular complication, 275/527 (52.2\%) for any macrovascular complication and 227/276 (82.2\%) for any complication.

Conclusion: The high burden of diabetes in Melbourne hospital inpatients has major implications for patient health and health care expenditure. Optimising care of these high-risk patients has the potential to decrease inpatient morbidity and length of stay as well as preventing or delaying future complications. A formal Australian national audit of inpatient diabetes would determine its true prevalence and consequences, allowing rational planning to deal with shortcomings in its management.

Patients with diabetes were asked about duration and type of diabetes, treatment, medical management, diabetes and dietetic education, surveillance and prevalence of complications, and smoking.

Hospital data are shown separately except for Eastern Health which includes Box Hill, Maroondah and Angliss Hospitals. Ten of Melbourne's 19 adult general hospitals participated, including four of five major tertiary teaching hospitals (The Alfred, Austin, Monash and St Vincent's) as well as six other teaching and community hospitals (Eastern, Sandringham, Sunshine and Western). One of eight rehabilitation hospitals also participated (Caulfield).

To validate self-reported diabetes diagnoses, diabetes status was confirmed in medical records of $10 \%$ of patients in each hospital. Three patients were misclassified (two without and one with diabetes), indicating an accuracy of $98.7 \%$. Self-reported admission diagnoses matched those on the discharge summary in $90 \%$ $95 \%$ of a subset of patients.

\section{Statistics}

Continuous data are shown as mean $(95 \% \mathrm{CI})$. Unpaired $t$ tests or one-way analyses of variance were used to compare groups as appropriate. For categorical data, proportions were analysed using $\chi^{2}$ tests with continuity correction where appropriate, followed by unadjusted $z$ tests to compare individual proportions. Sandringham was excluded from some prevalence ranges for individual hospitals as only eight patients had diabetes. Binomial exact methods were used to calculate $95 \%$ confidence intervals for proportions. Pearson correlation between community and 
1 Diabetes prevalence $(95 \% \mathrm{Cl})$ in inpatients at Melbourne metropolitan hospitals

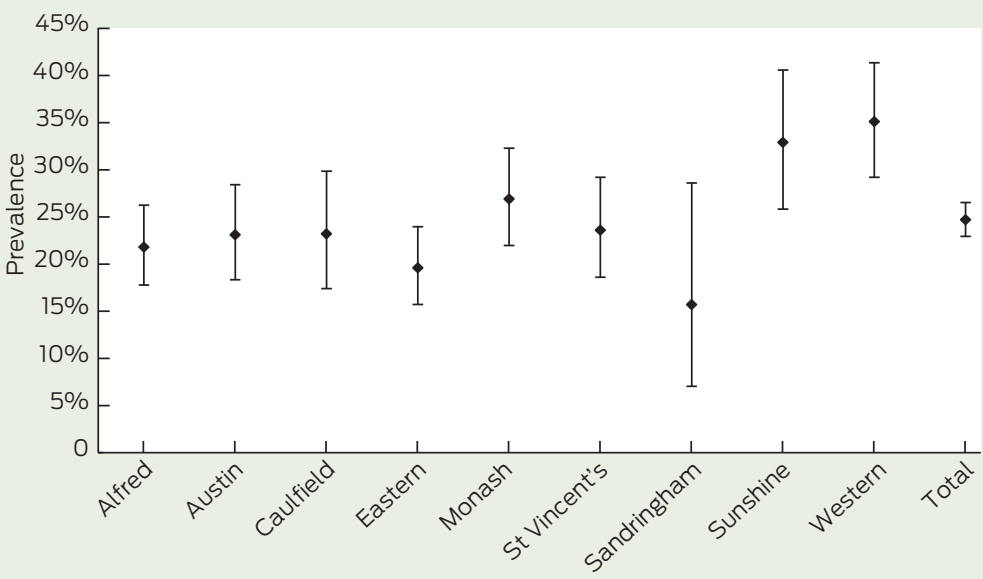

Hospital

hospital prevalence of diabetes was calculated. Analyses were performed using IBM SPSS Statistics version 20.

\section{Results}

Of 2311 patients approached, three patients declined to participate and the remaining 2308 were interviewed or their medical records were used. Data were $69.4 \%-85.6 \%$ complete for individual questions apart from diabetes diagnosis (98.5\%). Additionally, weight was available for 1604 (69.5\%) and age for 583 patients (25.3\%). Data from patients with diabetes were $61.6 \%-100.0 \%$ complete for individual questions. Diabetes status was obtained in 2273 of 2308 inpatients $(98.5 \%)$. Of these, $562(24.7 \%)$ had diabetes (95\% CI, $22.9 \%-26.5 \%)$, with hospital prevalences ranging from $15.7 \%$ to $35.1 \%$ (Box $1, P<0.001$ ).

\section{Associations with diabetes}

A family history of diabetes was noted in 174 of 323 patients with diabetes (53.9\%) compared with 356 of 1354 patients without diabetes $(26.3 \%)(P<0.001)$. Patients with diabetes were more likely to be over 40 years old $(94.3 \%$ v $80.6 \%, P<0.001)$ and were older than patients without diabetes (mean age, 72.4 years; $95 \%$ CI, 70.3-74.4 years v 67.5 years; $95 \%$ CI, 65.6-69.4 years; $n=583 ; P=0.001$ ). Patients with diabetes were heavier (mean weight, $80.8 \mathrm{~kg}$; 95\% CI, 78.8$82.7 \mathrm{~kg}$ v $73.6 \mathrm{~kg} ; 95 \% \mathrm{CI}, 72.6-74.6 \mathrm{~kg}$; $P<0.001)$, and more likely to be taking lipid-lowering $(60.6 \%$ v $28.3 \%$,
$P<0.001)$, antihypertensive $(67.7 \% \mathrm{v}$ $44.6 \%, P<0.001)$ and blood-thinning medication $(60.2 \% \mathrm{v} 40.8 \%, P<0.001)$. Surprisingly, glucocorticoid use was similar in patients with and without diabetes $(18.9 \%$ v $15.2 \%, P=0.10)$.

\section{Patients with diabetes}

Mean duration of diabetes was 11.9 years (95\% CI, 10.9-13.0 years) with no difference between hospitals $(n=334$; $P=0.62)$. Of 474 patients with recorded diabetes type, $91.8 \%$ had type 2 diabetes (range between hospitals, 83.3\%-98.1\%; lowest, The Alfred; highest, Western and Sunshine; $P=0.001)$ and $4.9 \%$ had type 1 diabetes (range, $0-8.6 \%$ ). Cystic fibrosis-induced diabetes accounted for five cases (1.1\%) and glucocorticoidinduced diabetes accounted for seven $(1.5 \%)$. It is likely that glucocorticoidinduced diabetes was underreported since patients may not recognise the link between glucocorticoid use and diabetes.

Admission diagnosis was recorded in 404 patients with diabetes. Of these, only four had a diagnosis directly related to problems with glycaemic control. Cardiovascular disease accounted for 59 admissions. Of 82 patients admitted with infections, 21 had respiratory infections, 17 had skin and/or foot infections and 10 had genitourinary infections. Forty patients were admitted for diabetes-related surgery (peripheral vascular disease surgery, foot surgery, coronary artery bypass grafting, endarterectomy or renal failure-related surgery) and 71 for surgery unrelated to diabetes. Five admissions related to transplantation, and other indications accounted for the remaining 147.

Complete information on diabetes medication was available in 388 patients. Oral hypoglycaemic agents were used by 270 patients $(69.6 \%$; range, $58.2 \%-86.8 \%, P<0.001$; lowest, The Alfred; highest, Sunshine). Insulin was used by 158 patients (40.7\%; range, $31.8 \%-55.3 \%, P=0.12$ ), 91 with oral agents $(23.5 \%$; range, $13.6 \%-42.1 \%, \quad P<0.001$; lowest, Eastern; highest, Sunshine) and 67 without oral agents $(17.3 \%)$. The remaining 51 patients $(13.1 \%)$ were not recorded as taking any diabetic medication.

In 227 patients with relevant information, 96 (42.3\%) took metformin alone, $54(23.8 \%)$ a sulfonylurea alone, and $57(25.1 \%)$ took both, with no difference between hospitals $(P=0.32)$. The remaining 20 patients took thiazolidinediones, dipeptidyl peptidase 4 (DPP4) inhibitors or acarbose alone or in combination with metformin or a sulfonylurea. Insulin delivery was noted in 189 patients; 168 used pens (88.9\%; range, $64.3 \%-96.6 \%, P=0.06)$, $18(9.5 \%)$ used syringes and three (1.6\%) used a pump.

Of 495 patients, $331(66.9 \%)$; range, $50.0 \%-80.5 \%, P=0.006$; highest Western, Sunshine) were managed by their general practitioners and three $(0.6 \%)$ had no medical input; the remainder were managed by specialists in private practice or in outpatient clinics. Diabetes education was reported in 258 of 424 patients $(60.8 \%$; range, $50.0 \%-72.1 \%, P=0.48)$ and 241 of 437 patients (55.1\%; range, $49.3 \%-$ $83.3 \%, P=0.005$; lowest, Sunshine; highest, Caulfield) had seen a dietitian. Most patients monitored home blood glucose levels (347/427, 81.3\%; range, $66.7 \%-91.9 \%, P=0.03$; lowest, The Alfred; highest, Eastern).

\section{Complications surveillance}

Eye checks were reported in 360 of 449 patients, $(80.2 \%$; range, $69.2 \%-100 \%$, $P=0.59)$ whereas only $62.9 \%$ recalled urine testing to assess kidney damage (range, $13.0 \%-82.1 \%, P<0.001$; lowest, St Vincent's; highest, Caulfield). Foot care in the previous year was reported in 296 of 451 patients $(65.6 \%$; range, $50 \%-69.8 \%, P=0.42)$. Two hundred 
2 Prevalence $(95 \% \mathrm{Cl})$ of any microvascular or macrovascular complication in inpatients with diabetes at Melbourne metropolitan hospitals

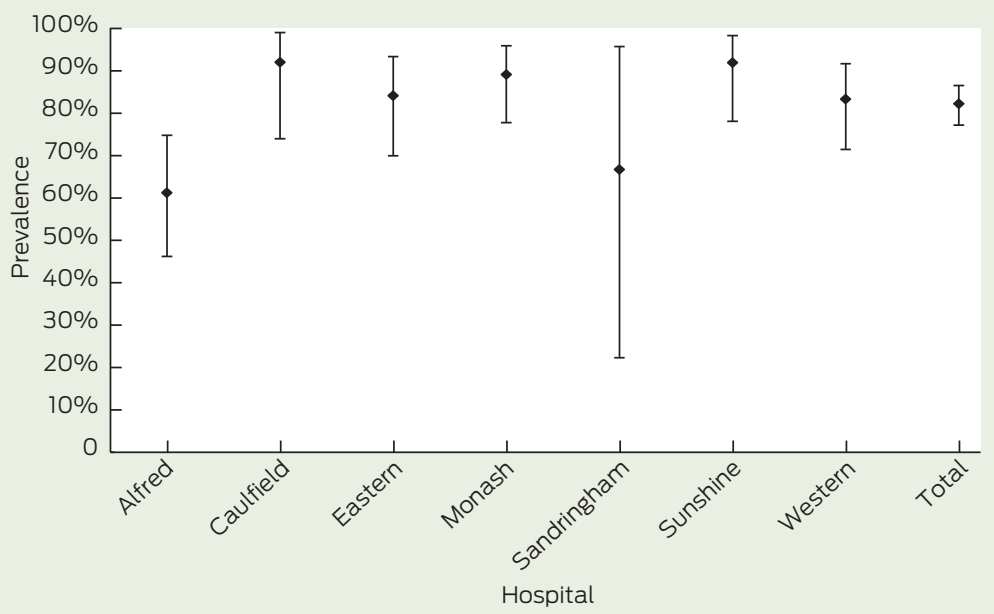

and fifty-seven of 474 patients $(54.2 \%$; range, $33.3 \%-61.9 \%, P=0.63$ ) had ever smoked, whereas 38 of 483 patients (7.9\%; range, $1.9 \%-13.7 \%, P=0.13$ ) were current smokers.

The prevalence of microvascular complications was high, with 207 of 290 patients $(71.4 \%$; range, $61.8 \%-$ $78.9 \%, P=0.43$ ) reporting at least one complication. Kidney damage was reported in 113 of 278 patients $(40.6 \%$; range, $26.4 \%-53.4 \%, P=0.05$; lowest, Western; highest, Monash), and eye damage was reported in 112 of 271 (41.3\%; range, $28.3 \%-60.7 \%, P=0.11$ ). Nerve damage was reported in 102 of 346 patients $(29.5 \%$; range, $23.3 \%-$ $44.1 \%, P=0.43)$.

Macrovascular complications were also highly prevalent, with 275 of 527 patients (52.2\%; range, $43.4 \%-60.9 \%$, $P=0.42$ ) reporting at least one complication. Myocardial infarction, angina or coronary artery graft surgery were reported in 197 of 500 patients (39.4\%; range, $16.7 \%-47.4 \%, P=0.24$ ). Stroke or transient ischaemic attack were reported in 99 of 490 patients (20.2\%; range, $10.0 \%-25.3 \%, P=0.63$ ), and 90 of 473 patients $(19.0 \%$; range, $16.4 \%-25.7 \%, P=0.81$ ) had angioplasty or bypass surgery for peripheral vascular disease.

Sixty-three of 346 patients (18.2\%; range, $8.9 \%-22.7 \%, P=0.53)$ reported a history of foot ulcer, and 53 of 401 patients $(13.2 \%$; range, $4.3 \%-23.5 \%$, $P=0.04$; lowest, Sunshine; highest, Caulfield) had a current ulcer. Overall, 227 of 276 patients (82.2\%) with complete information in seven hospitals reported at least one microvascular or macrovascular complication (Box 2).

\section{Discussion}

The major finding of this study is that one in four inpatients in Melbourne hospitals had self-reported diabetes. Patients with diabetes were older and heavier than those without, and were more likely to be treated for other cardiovascular risk factors including hypertension and dyslipidaemia. Not surprisingly, most patients had type 2 diabetes, and mean duration was 11.9 years. Almost half were treated with insulin alone or in combination with oral hypoglycaemic agents. More than $80 \%$ of patients had at least one microvascular or macrovascular complication. These findings show that inpatients with diabetes are complex cases and have significant comorbid conditions.

There are currently no reports of Australian inpatient diabetes prevalence, and this study fills that knowledge gap. The $26.2 \%$ prevalence of known diabetes in a single US hospital $^{5}$ is similar to our finding. The most recent International Diabetes Federation estimates for diabetes prevalence in the adult community are $10.0 \%$ for Australia and $10.9 \%$ for the US. ${ }^{8}$ In a UK survey of 206 centres, patients with diabetes accounted for $5.8 \%-25.8 \%$ of inpatients with a median of about $15 \%,{ }^{6}$ compared with a community prevalence of $6.6 \%{ }^{8}$ A study of over 2 million Japanese inpatients found an $11.4 \%$ prevalence of type 2 diabetes ${ }^{7}$ compared with a community prevalence of $7.6 \%{ }^{8}$

The prevalence of diagnosed diabetes in the Victorian community is $5.1 \%$ as assessed by National Diabetes Supply Scheme (NDSS) registration, ${ }^{9}$ although this is an underestimate that excludes unregistered and undiagnosed patients. Rates within the Melbourne metropolitan area range from $2.4 \%$ in the Inner Melbourne local government area to $6.7 \%$ in Brimbank (centred on Sunshine) and Greater Dandenong. Diabetes prevalence was calculated in the four local government areas nearest each hospital. Assuming these account for most admissions, there was a strong correlation between inpatient and community prevalence of diabetes $\left(r^{2}=0.60\right.$, $P=0.01$ ) (Box 3). Even assuming that $50 \%$ of diabetes in the community is undiagnosed, ${ }^{1}$ these figures suggest a threefold increased hospitalisation risk with diabetes. Factors accounting for the remaining variance between hospitals may include differences in education, social class, ethnicity, provision of community diabetes support services and inpatient services available in each hospital.

Sunshine and Western Hospitals are located among the most disadvantaged areas in Melbourne, while Alfred, Caulfield and Sandringham Hospitals are in advantaged areas. ${ }^{10}$ Consistent with the NDSS data, people living in the most disadvantaged areas have diabetes rates that are twice those in the most advantaged.11 Diabetes rates are higher in populations from Asian backgrounds than those from southern European and UK backgrounds, ${ }^{11}$ and $>50 \%$ of the residents served by Western and Sunshine Hospitals were born overseas, mainly in Asia, with up to $60 \%$ speaking a language other than English at home. ${ }^{12}$

There was a significant difference in medical management between hospitals, with more Western and Sunshine Hospital patients being managed by their GPs than those at other hospitals. There are no published data examining the proportion of patients with diabetes who are managed solely by GPs. However, long-term data suggest that increased exposure to specialist care lowers the 


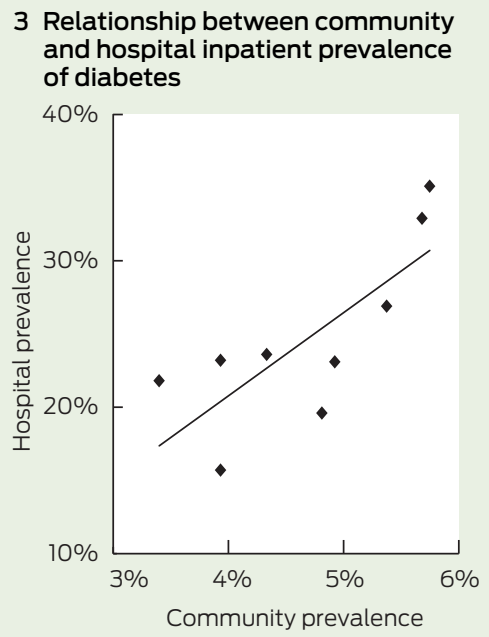

risk of complications in patients with type 1 diabetes. ${ }^{13}$ Further, limited local data suggest glycated haemoglobin $\left(\mathrm{HbA}_{1 \mathrm{c}}\right)$ is significantly improved in patients managed by advanced skill GPs (those who have taken undertaken postgraduate training in advanced diabetes care) supported by an endocrinologist and diabetes educator. ${ }^{14}$ However, only $55 \%-61 \%$ of patients in our study recall being seen by a diabetes educator and/or dietitian, suggesting an opportunity to optimise ambulatory diabetes care.

A high proportion of inpatients had complications, which may reflect their older age and long duration of diabetes. Given that diabetes is the leading cause of end-stage kidney disease, ${ }^{15}$ it is surprising that only $63 \%$ of patients recalled having a urine test to assess kidney damage, although this may reflect recall bias. Eighty per cent of inpatients had their eyes tested and $66 \%$ had their feet checked. Hospital admission provides an opportunity to optimise glycaemic control thereby reducing microvascular complications. ${ }^{16}$ Macrovascular complications were also common, being found in $52 \%$ of patients. Since the presence of macrovascular disease increases the risk of future major vascular events threefold in patients with diabetes, ${ }^{17}$ hospital admission is also an opportunity to identify and treat these high-risk patients. For example, the admission may be an opportunity to optimise management of diabetes and other cardiovascular risk factors in these patients.
It is estimated that patients with diabetes account for $26 \%$ of inpatient expenditure in the US (US\$123 billion in 2012), ${ }^{4}$ but there are no equivalent Australian data. Australian Institute of Health and Welfare (AIHW) 2010-11 data suggest that diabetes accounted for $2.5 \%$ of all hospitalisations in Australia, ${ }^{18}$ which is considerably lower than our finding. AIHW data include day cases, comprising $58 \%$ of admissions, ${ }^{19}$ which are likely to dilute the prevalence of diabetes. Additionally, there were changes to diabetes coding from 2008-2012 that decreased prevalence by $63 \% .{ }^{19}$ These factors suggest that the AIHW result is a gross underestimate. Interestingly, the coding change has been reversed so that it is now mandated that diabetes always be coded irrespective of admission diagnosis.

Compounding its high prevalence, patients with diabetes have longer hospital stays than those without. Length of stay for patients with diabetes was 2.8 days longer than for those without, irrespective of age, socioeconomic status, admission type or case complexity in a large English database. ${ }^{20}$ Further, diabetes was independently associated with excess bed use, especially for surgical admissions. We also found that general medical inpatients with known diabetes stayed in hospital for 1 day longer than normoglycaemic patients without diabetes. ${ }^{21}$

The major strength of this study is that it encompasses $>2000$ patients across a wide range of metropolitan locations. Although self-reporting of diabetes may be perceived as a weakness, cross-checking of medical records showed $98.7 \%$ accuracy. Nevertheless, the study has some limitations. Length of stay, outcomes and biochemical data including $\mathrm{HbA}_{1 \mathrm{c}}$ that may now be used to diagnose diabetes ${ }^{22}$ were not recorded. Indeed, type 2 diabetes is commonly undiagnosed in the community, ${ }^{1}$ so that the reported prevalence may be an underestimate. Hospitals were studied over a 2-year period, although each survey was completed in a single day. Importantly, there was no relationship between study date and diabetes prevalence across hospitals. Finally, patient numbers at Sandringham were small, resulting in wide confidence intervals, and the inpatient mix in some hospitals may have biased results.

There are significant gaps in diabetes education and complications surveillance, and hospital admission provides an opportunity to fill these gaps. Although diabetes was not the primary reason for admission in most patients, these findings have significant implications for their short- and long-term outcomes. Appropriate management of diabetes early in the admission may shorten length of stay and decrease readmission rates. Further, optimising management of diabetes and its complications reduces the risk of morbidity due to long-term complications. These findings clearly have a bearing on Australian health expenditure. Ensuring that diabetes and its comorbid conditions are identified and managed appropriately may reduce inpatient costs in the present admission and also decrease future costs related to preventable diabetesrelated complications. It is time for a formal Australian national inpatient diabetes audit that ideally captures biochemical data to determine its true prevalence and consequences. Such an audit would allow a comprehensive assessment of this high-risk group and permit rational planning to deal with shortcomings in their management.

Acknowledgements: The authors would like to thank Ghazaleh Ahmadi (Eastern Health) and Alan McCubbin (Austin Health) for assistance with collating data. We would also like to thank the numerous dietitians, diabetes nurse educators, hospital medical officers, registrars, endocrinologists and medical students who assisted in data collection.

Competing interests: No relevant disclosures.

Received 22 Nov 2013, accepted 3 Jun 2014.

1 Dunstan DW, Zimmet PZ, Welborn TA, et al. The rising prevalence of diabetes and impaired glucose tolerance: the Australian Diabetes, Obesity and Lifestyle Study. Diabetes Care 2002; 25: 829-834.

2 Australian Institute of Health and Welfare. Diabetes prevalence in Australia: an assessment of national data sources. Canberra: AlHW, 2009. (AlHW Cat. No. CVD 46.) http://www.aihw.gov.au/publicationdetail/?id=6442468288 (accessed Jun 2014).

3 National Diabetes Services Scheme. All types of diabetes. Statistical snapshot. 2013 http:// www.ndss.com.au/Global/Data Snapshots/ December 2013/NDSS-StatisticalSnapshot31December-v1401_AllTypes.pdf (accessed Jun 2014).

4 American Diabetes Association. Economic costs of diabetes in the US in 2012. Diabetes Care 2013; 36: 1033-1046. 
5 Umpierrez GE, Isaacs SD, Bazargan N, et al. Hyperglycemia: an independent marker of inhospital mortality in patients with undiagnosed diabetes. J Clin Endocrinol Metab 2002; 87: 978-982.

6 National Health Service. National Diabetes Inpatient Audit (NaDIA) - 2011. Key findings about the quality of care of inpatients with diabetes in England and Wales. Leeds, UK: Heath and Social Care Information Centre, 2012. http://www.hscic.gov.uk/catalogue/ PUB06279/nati-diab-inp-audi-11-nat-rep.pdf (accessed Jun 2014).

7 Kubo T, Fujino Y, Murata A, et al. Prevalence of type 2 diabetes among acute inpatients and its impact on length of hospital stay in Japan. Intern Med 2011; 50: 405-411.

8 International Diabetes Federation. IDF Diabetes Atlas. 6th ed. Brussels, Belgium: IDF, 2013. http://www.idf.org/diabetesatlas (accessed Jun 2014).

9 National Diabetes Services Scheme. Australian Diabetes Map 2013. http://www.ndss.com.au/ en/Australian-Diabetes-Map/Map (accessed Oct 2013).

10 Australian Bureau of Statistics. Census of Population and Housing: Socio-Economic Indexes for Areas (SEIFA), Australia, 2011. (ABS Cat. No. 2033.0.55.001.) http://www.abs.gov.
au/ausstats/abs@.nsf/DetailsPage/2033.0.55. 0012011?OpenDocument (accessed Sep 2013).

11 Australian Bureau of Statistics. Australian Health Survey: Updated Results, 2011-2012. Canberra: ABS, 2013. (ABS Cat. No.

4364.0.55.003.) http://www.abs.gov.au/ ausstats/abs@.nsf/Lookup/1A8F3DE217DE1057 CA257B82001792F4?opendocument (accessed Sep 2013).

12 Australian Bureau of Statistics. National Regional Profile 2007-2011. Canberra: ABS, 2013. http://www.ausstats.abs.gov.au/ ausstats/nrpmaps.nsf/NEW+GmapPages/ national+regional+profile (accessed Sep 2013).

13 Zgibor JC, Songer TJ, Kelsey SF, et al. Influence of health care providers on the development of diabetes complications: long-term follow-up from the Pittsburgh Epidemiology of Diabetes Complications Study. Diabetes Care 2002; 25: 1584-1590.

14 Jackson C, Tsai J, Brown C, et al. GPs with special interests - impacting on complex diabetes care. Aust Fam Physician 2010; 39: 972-974.

15 Molitch ME, DeFronzo RA, Franz MJ, et al Nephropathy in diabetes. Diabetes Care 2004; 27 Suppl 1: S79-S83.

16 UK Prospective Diabetes Study (UKPDS) Group. Intensive blood-glucose control with sulphonylureas or insulin compared with conventional treatment and risk of complications in patients with type 2 diabetes (UKPDS 33). Lancet 1998; 352: 837-853.

17 Collins R, Armitage J, Parish S, et al. MRC/BHF Heart Protection Study of cholesterol-lowering with simvastatin in 5963 people with diabetes: a randomised placebo-controlled trial. Lancet 2003; 361: 2005-2016.

18 Australian Institute of Health and Welfare. Health service use 2013. http://www. aihw.gov.au/diabetes/health-serviceuse/\#hospitalisations (accessed Mar 2014).

19 Australian Institute of Health and Welfare. Australian hospital statistics 2011-12. Canberra: AlHW, 2013. (AlHW Cat. No. HSW 134; Health Services Series 50.) http://www.aihw.gov.au/ publication-detail/?id=60129543133 (accessed Jun 2014).

20 Whitston M, Chung S, Henderson J, Young B. What can be learned about the impact of diabetes on hospital admissions from routinely recorded data? Diabet Med 2012; 29: 1199-1205.

21 Baker ST, Chiang CY, Zajac JD, et al. Outcomes for general medical inpatients with diabetes mellitus and new hyperglycaemia. Med J Aust 2008; 188: 340-343.

22 American Diabetes Association. Standards of medical care in diabetes -- 2013. Diabetes Care 2013; 36 Suppl 1: S11-S66. 